\title{
Partially Overlapped Channel Assignment for Multi-Channel Wireless Mesh Networks
}

\author{
A. Hamed Mohsenian Rad and Vincent W.S. Wong \\ Department of Electrical and Computer Engineering \\ The University of British Columbia, Vancouver, Canada \\ e-mail: $\{$ hamed, vincentw $\} @$ ece.ubc.ca
}

\begin{abstract}
The aggregate capacity of wireless mesh networks can be increased by the use of multiple frequency channels and multiple network interface cards in each router. Recent results have shown that the performance can further be increased when both non-overlapped and partially overlapped channels are being used. In this paper, we propose a linear model for a joint channel assignment, interface assignment, and scheduling design. We propose the channel overlapping matrix and mutual interference matrices to model the non-overlapped and partially overlapped channels. Since the model is formulated as a linear mixedinteger program with a few integer variables, the computation complexity is low and it is feasible for implementation. Simulation results show that the aggregate network capacity increases by $90 \%$ when all partially overlapped channels within the $802.11 \mathrm{~b}$ frequency band are being used.
\end{abstract}

\section{INTRODUCTION}

A multi-interface multi-channel wireless mesh network (MIMC-WMN) consists of a number of stationary wireless routers, forming a multi-hop wireless backbone. Each router is equipped with multiple network interface cards (NICs) [1]. Each NIC operates on a distinct frequency channel in the IEEE $802.11 \mathrm{a} / \mathrm{b} / \mathrm{g}$ bands. The wireless mesh routers serve as access points for wireless mobile devices. Some of them also act as gateways to the Internet via high-speed wired links. Two neighboring routers can establish a logical link if each one has an NIC operating on the same channel. A sample MIMCWMN is illustrated in Fig. 1.

Within the IEEE 802.11 frequency bands, the number of available channels is limited. For example, the IEEE $802.11 \mathrm{~b} / \mathrm{g}$ standards have 11 channels, of which 3 channels are nonoverlapped. This implies that some logical links (e.g., links $(a, b)$ and $(f, h)$ in Fig. 1) may be assigned to the same channel. Interference can occur if these links are close to each other. The channel assignment problem determines which frequency channel should be allocated to each logical link.

The number of available NICs in each router is also limited. In the experimental MIMC-WMN test-beds in [1] and [2], each mesh router is equipped with two NICs. Providing up to four NICs is also considered reasonable in [2], [3]. A small number of NICs implies that some logical links in a router may need to share an NIC to transmit and receive data packets (e.g., links $(e, a)$ and $(e, c)$ in Fig. 1). When two logical links in a router share an NIC, they cannot be active simultaneously. It significantly decreases their effective data rate. The interface assignment problem determines which

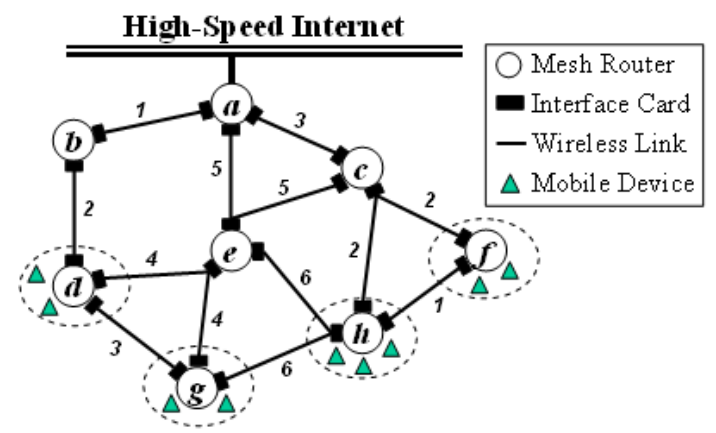

Fig. 1. An MIMC-WMN backbone with eight mesh routers, six frequency channels, and three NICs per router. The number on each link indicates the operating channel number.

NICs should be assigned to each logical link. The channel assignment and interface assignment problems are related. Two links that share an NIC are required to operate over the same frequency channel [1].

Various channel assignment algorithms have been reported in the literature (e.g., [1]-[4]). Most of them only consider the assignment of non-overlapped (orthogonal) frequency channels. Recent results have shown that the network capacity can further be increased when both non-overlapped and partially overlapped channels are being used [5], [6]. A joint channel assignment and congestion control algorithm is proposed in [5]. The proposed algorithm uses a channel weighting matrix to model the overlapping between different channels. However, the model is non-linear which makes it difficult for implementation in practical WMNs. Mishra et al. extended the work in [3] and proposed an algorithm which assigns both non-overlapped and partially overlapped channels [6]. The algorithm is based on solving a discrete optimization problem. However, since heuristics are used to obtain the solutions, the solutions may not always be optimal [7].

In this paper, we systematically model a joint channel assignment, interface assignment, and scheduling design problem. The contributions of our work are as follows:

- Our proposed model takes into account various parameters including the number of available frequency channels, the number of available NICs in each wireless router, transmission power, path loss information, signal to interference plus noise ratio, expected traffic load, and frequency response of each channel filter. 
- Since the model is formulated as a linear mixed-integer program with a few integer variables, the computation complexity is low and it is feasible for implementation.

- We propose the channel overlapping matrix and mutual interference matrices to model the non-overlapped and partially overlapped channels.

- Simulation results show that the aggregate network capacity increases by $90 \%$ when all partially overlapped channels within the IEEE $802.11 \mathrm{~b}$ frequency band are being used.

The rest of the paper is organized as follows. The problem formulation is described in Section II. Our proposed joint design is presented in Section III. Performance evaluations are given in Section IV. Conclusions and future work are given in Section V.

\section{SySTEM MODEL}

Consider an MIMC-WMN and assume that $N$ denotes the set of stationary wireless mesh routers. Each router is equipped with $I$ NICs. There are $C$ frequency channels available. We assume that the network's logical topology has been predetermined. Let $L$ denote the set of all unidirectional logical links. The logical link from router $a$ to router $b$ is denoted by $(a, b) \in L$. We assume the connectivity to be symmetric. That is, link $(a, b) \in L$ if and only if $(b, a) \in L$.

\section{A. Channel Assignment Model}

For any two routers $a, b \in N$ such that there exists a logical link $(a, b) \in L$, we define a $C \times 1$ channel assignment vector $\overline{\mathbf{x}}_{a b}$. If router $a$ communicates with router $b$ over the $i^{\text {th }}$ frequency channel, then the $i^{t h}$ element in $\overline{\mathbf{x}}_{a b}$ is equal to 1 ; otherwise, it is equal to zero. As an example, assume that $C=$ 5 and router $a$ is assigned to communicate with router $b$ over the second channel. We have, $\overline{\mathbf{x}}_{a b}=\left[\begin{array}{lllll}0 & 1 & 0 & 0 & 0\end{array}\right]^{T}$.

To establish the logical link $(a, b) \in L$, routers $a$ and $b$ should assign a common frequency channel to communicate with each other. This requires that,

$$
\overline{\mathbf{x}}_{a b}=\overline{\mathbf{x}}_{b a}, \quad \forall a, b \in N, \quad(a, b) \in L
$$

and

$$
\mathbf{1}^{T} \overline{\mathbf{x}}_{a b}=1, \quad \forall a, b \in N, \quad(a, b) \in L
$$

where 1 denotes a $C \times 1$ vector with all entries equal to 1 . The term $\mathbf{1}^{T} \overline{\mathbf{x}}_{a b}$ is equal to 1 if router $a$ assigns one of the available frequency channels to communicate with router $b$.

Consider two logical links $(a, b),(c, d) \in L$. If they are assigned to the same channel, then the term $\overline{\mathbf{x}}_{a b}^{T} \overline{\mathbf{x}}_{c d}$ is equal to 1 ; otherwise, it is equal to 0 .

\section{B. Interface Assignment Model}

For any two routers $a, b \in N$ such that $(a, b) \in L$, we define an $I \times 1$ interface assignment vector $\overline{\mathbf{y}}_{a b}$. If the $i^{\text {th }}$ network interface in router $a$ is used to communicate with router $b$, then the $i^{t h}$ element in $\overline{\mathbf{y}}_{a b}$ is equal to 1 ; otherwise, it is equal to zero. As an example, assume that $I=3$ and router $a$ assigns its first network interface to communicate with router $b$. We have, $\overline{\mathbf{y}}_{a b}=\left[\begin{array}{ccc}1 & 0 & 0\end{array}\right]^{T}$. Note that in general, $\overline{\mathbf{y}}_{a b} \neq \overline{\mathbf{y}}_{b a}$.

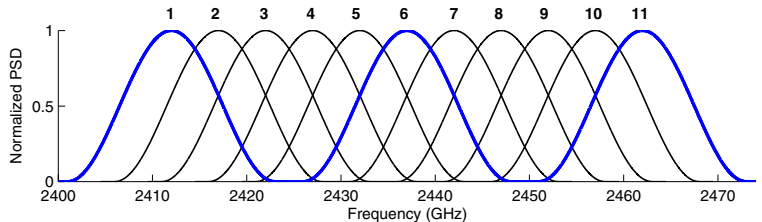

Fig. 2. Available eleven partially overlapped channels in $802.11 \mathrm{~b}$ frequency band. The number on each curve indicates the corresponding channel number. Channels 1, 6, and 11 are non-overlapped (orthogonal).

To establish the logical link $(a, b) \in L$, routers $a$ and $b$ should assign one of their NICs to communicate with each other. This requires that,

$$
\mathbf{1}^{T} \overline{\mathbf{y}}_{a b}=1, \quad \forall a, b \in N, \quad(a, b) \in L
$$

where 1 denotes an $I \times 1$ vector with all entries equal to 1 . The term $\mathbf{1}^{T} \overline{\mathbf{y}}_{a b}$ is equal to 1 if router $a$ assigns one of its NICs to communicate with router $b$.

Consider two logical links $(a, b),(a, c) \in L$ such that they share router $a$. If they are assigned to the same NIC, then the term $\overline{\mathbf{y}}_{a b}^{T} \overline{\mathbf{y}}_{a c}$ is equal to 1 ; otherwise, it is equal to 0 .

If two neighboring logical links $(a, b),(a, c) \in L$ are assigned to the same NIC (i.e., $\overline{\mathbf{y}}_{a b}^{T} \overline{\mathbf{y}}_{a c}=1$ ), then they will be assigned the same frequency channel (i.e., $\overline{\mathbf{x}}_{a b}^{T} \overline{\mathbf{x}}_{a c}=1$ ). On the other hand, if they are not assigned to the same NIC (i.e., $\overline{\mathbf{y}}_{a b}^{T} \overline{\mathbf{y}}_{a c}=0$ ), then they will be assigned to two different frequency channels (i.e., $\overline{\mathbf{x}}_{a b}^{T} \overline{\mathbf{x}}_{a c}=0$ ). We can model the relationship between the channel assignment and the interface assignment vectors to be as follows:

$$
\overline{\mathbf{x}}_{a b}^{T} \overline{\mathbf{x}}_{a c}=\overline{\mathbf{y}}_{a b}^{T} \overline{\mathbf{y}}_{a c}, \quad \forall a, b, c \in N, \quad(a, b),(a, c) \in L .
$$

\section{Channel Overlapping Matrix}

Assume that $m$ and $n$ are two of the available channels within the 802.11 frequency band (i.e., $m, n \in\{1, \cdots C\}$ ). Let $F_{m}(\omega)$ and $F_{n}(\omega)$ denote the power spectral density (PSD) functions of the band-pass filters for channels $m$ and $n$, respectively. The PSD functions can be obtained from the channels' frequency responses. Without loss of generality, we assume the use of raised cosine filters [8]. Fig. 2 shows the frequency responses of the channel filters in the IEEE $802.11 \mathrm{~b}$ frequency band. To model the overlapping among different channels, we define a symmetric $C \times C$ channel overlapping matrix $\mathbf{W}$. The entry in the $m^{\text {th }}$ row and the $n^{\text {th }}$ column of $\mathbf{W}$ is denoted by scalar $w_{m n}$ and is defined to be as follows:

$$
w_{m n}=\frac{\int_{-\infty}^{\infty} F_{m}(\omega) F_{n}(\omega) d \omega}{\int_{-\infty}^{\infty} F_{m}^{2}(\omega) d \omega} .
$$

Now assume that channels $m$ and $n$ are assigned to arbitrary links $(a, b)$ and $(c, d)$, respectively. Let $p_{a}$ denote the transmission power of router $a$. Also let $g_{a d}$ denote the path loss from router $a$ to router $d$. The interference power from link $(a, b)$ on link $(c, d)$ can be modeled as:

$$
\overline{\mathbf{x}}_{a b}^{T} \mathbf{W} \overline{\mathbf{x}}_{c d} g_{a d} p_{a}=w_{m n} g_{a d} p_{a} .
$$




\section{Mutual Interference Model}

We first consider a MIMC-WMN where only nonoverlapped channels are being used, two links $(a, b),(c, d) \in L$ are defined to be mutually interfered with each other whenever they are assigned to the same channel (i.e., $\overline{\mathbf{x}}_{a b}^{T} \overline{\mathbf{x}}_{c d}=1$ ) and the sender/receiver of one link is within the interference range of the sender/receiver of the other link. The interference range is then defined as the region where a given receiver cannot decode the signal correctly if there is another transmission within that range. Given the modulation scheme, the interference range depends on the minimum required signal to interference plus noise ratio SINR $_{\min }$.

Now consider an MIMC-WMN where both non-overlapped and partially-overlapped channels are being used. Two neighboring links $(a, b),(c, d) \in L$ are assigned to channels $m$ and $n$, respectively. If the interference power of the transmission on link $(a, b)$ causes the signal to interference plus noise ratio on link $(c, d)$ to be below $\operatorname{SINR}_{\min }$, then the transmitter of link $(a, b)$ is within the interference range of the receiver of link $(c, d)$. That is,

$$
\frac{g_{c d} p_{c}}{w_{m n} g_{a d} p_{a}+\eta}<S_{\text {INR }} R_{\text {min }}
$$

where $\eta$ denotes the thermal noise power. Without loss of generality, we model the path loss $g_{a d}$ using the Friis free space model [8]:

$$
g_{a d}=\frac{\alpha}{\left(r_{a d}\right)^{\kappa}}
$$

where $r_{a d}$ is the Euclidean distance between routers $a$ and $d, \kappa$ is the path loss exponent, and $\alpha$ is a constant which depends on the transmitter and receiver antenna gains and signal wavelength. By substituting (8) into (7) and re-arranging the terms, link $(a, b)$ interferes with link $(c, d)$ then,

$$
r_{a d}<\sqrt[\kappa]{\left(\frac{\alpha p_{a}}{g_{c d} p_{c} / S I N R_{m i n}-\eta}\right) w_{m n}}
$$

The importance of (9) is that we now have different interference ranges depending on the assigned channels to the neighboring links. The less the frequency overlapped, the shorter the interference range is. Given that the bandwidth and roll-off factor are the same in all raised cosine channel filters, the interference range only depends on the frequency channel separation $(|m-n|)$. This fact is illustrated in Fig. 3. The outermost circle indicates the interference range of receiver router $d$ when $|m-n|=0$ (i.e., the same channel is being assigned to links $(a, b)$ and $(c, d)$. The next circle shows the interference range when $|m-n|=1$. The innermost circle corresponds to the interference range when $|m-n|=3$. When $|m-n|>3$, there is no overlap between frequency channels $m$ and $n$ for IEEE $802.11 \mathrm{~b}$ (see Fig. 2a). Thus, the corresponding interference ranges are equal to zero. Note that in this example, transmission on link $(a, b)$ interferes with transmission on link $(c, d)$ only when either $|m-n|=0$ or $|m-n|=1$.

For any two links $(a, b),(c, d) \in L$, we define a symmetric $C \times C$ mutual interference matrix $\mathbf{M}_{c d}^{a b}$. If we have either

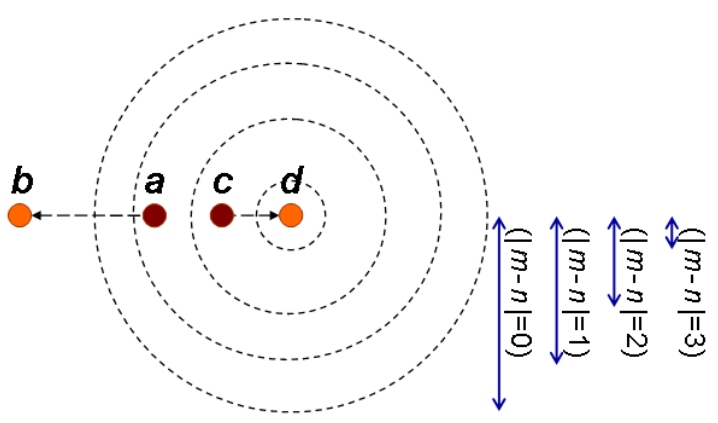

Fig. 3. Different interference ranges depending on the frequency channel separation $|m-n|$. Logical links $(a, b)$ and $(c, d)$ use channels $m$ and $n$ in $802.11 \mathrm{~b}$ frequency band, respectively.

$$
r_{a d}<\sqrt[\kappa]{\left(\frac{\alpha p_{a}}{g_{c d} p_{c} / S I N R_{\min }-\eta}\right) w_{m n}}
$$

or

$$
r_{c b}<\sqrt[\kappa]{\left(\frac{\alpha p_{c}}{g_{a b} p_{a} / S I N R_{\min }-\eta}\right) w_{n m}}
$$

then the entry in $m^{t h}$ row and $n^{t h}$ column of $\mathbf{M}_{c d}^{a b}$ is equal to 1 ; otherwise, it is equal to 0 . If the transmission powers are fixed, the mutual interference matrices are constant for stationary MIMC-WMNs. For the scenario in Fig. 3, the corresponding mutual interference matrices are tridiagonal with all diagonal, subdiagonal, and superdiagonal entries equal to one:

$$
\mathbf{M}_{c d}^{a b}=\mathbf{M}_{a b}^{c d}=\left[\begin{array}{cccccc}
1 & 1 & 0 & 0 & \cdots & 0 \\
1 & 1 & 1 & 0 & \cdots & 0 \\
0 & 1 & \ddots & \ddots & \ddots & \vdots \\
\vdots & \ddots & \ddots & \ddots & 1 & 0 \\
0 & \cdots & 0 & 1 & 1 & 1 \\
0 & \cdots & 0 & 0 & 1 & 1
\end{array}\right]_{11 \times 11}
$$

Note that if logical links $(a, b)$ and $(c, d)$ are far enough from each other, then all entries of $\mathbf{M}_{c d}^{a b}$ become zero. According to the definitions of channel assignment vectors and mutual interference matrices, we have:

$$
\overline{\mathbf{x}}_{a b}^{T} \mathbf{M}_{c d}^{a b} \overline{\mathbf{x}}_{c d}= \begin{cases}1, & \begin{array}{l}
\text { if links }(a, b) \text { and }(c, d) \text { are } \\
\text { mutually interfered },
\end{array} \\
0, & \text { otherwise. }\end{cases}
$$

\section{E. Link-Layer Flow Rates}

Let $0 \leq f_{a b} \leq 1$ denote the normalized link-layer flow rate on logical link $(a, b) \in L$ (i.e., the proportion of time that link $(b, a)$ is active). If two logical links $(a, b),(c, d) \in L$ are mutually interfered (i.e., $\overline{\mathbf{x}}_{a b}^{T} \mathbf{M}_{c d}^{a b} \overline{\mathbf{x}}_{c d}=1$ ), they cannot be active simultaneously. We can extend the interference constraint in [3] to have:

$$
f_{a b}+\sum_{c, d:(c, d) \in L} \overline{\mathbf{x}}_{a b}^{T} \mathbf{M}_{c d}^{a b} \overline{\mathbf{x}}_{c d} f_{c d} \leq 1 .
$$

Let $c^{0}$ denote the nominal data transmission rate (e.g., 11 Mbps data rate in IEEE 802.11b). The effective link-layer data rate on link $(a, b)$ is equal to $f_{a b} c^{0}$. 


\section{Joint Channel Assignment, Interface Assignment, AND SCHEduling Problem}

The mathematical formulation introduced in (1)-(12) models the channel assignment, interface assignment, mutual interference among the links, and link-layer flow rates when all non-overlapped and partially overlapped channels within the IEEE 802.11 frequency band are being used. The model can be employed to develop different cross-layer design schemes for MIMC-WMNs. In this paper, we focus on a joint channel assignment, interface assignment, and scheduling design problem. We assume that the network logical topology and routing paths have been pre-determined. For future work, we plan to relax these assumptions by integrating logical topology design and routing algorithms (see also [9]).

\section{A. Nonlinear Problem}

Given the expected end-to-end traffic rates and routing paths, we can determine the expected aggregated traffic load on each logical link $(a, b) \in L$. It is denoted by $\gamma_{a b}$. The link utilization on logical link $(a, b)$ is defined as the total traffic load $\gamma_{a b}$ divided by the effective link-layer data rate $f_{a b} c^{0}$. Based on the results from queuing theory, when the link utilization is close to 1 , the queueing delay tends to be very large [10]. On the other hand, a small value of the link utilization tends to provide a small queueing delay. It also implies that the network is less prone to congestion. Thus, our objective function is to minimize the maximum (i.e., bottleneck) link utilization in an attempt to manage the network capacity according to the expected traffic load. Given the parameters $I, C, L, \gamma_{a b}, c^{0}$, and $\mathbf{M}_{c d}^{a b}$ for all $a, b, c, d \in N$ such that $(a, b),(c, d) \in L$,

$$
\begin{aligned}
& \underset{\overline{\mathbf{x}}, \overline{\mathbf{y}}, f}{\operatorname{minimize}} \underset{a, b:(a, b) \in L}{\operatorname{maximum}} \frac{\gamma_{a b}}{f_{a b} c^{0}} \\
& \text { subject to } \\
& \overline{\mathbf{x}}_{a b}=\overline{\mathbf{x}}_{b a}, \\
& \mathbf{1}^{T} \overline{\mathbf{x}}_{a b}=1 \\
& \mathbf{1}^{T} \overline{\mathbf{y}}_{a b}=1 \\
& \overline{\mathbf{x}}_{a b}^{T} \overline{\mathbf{x}}_{a c}=\overline{\mathbf{y}}_{a b}^{T} \overline{\mathbf{y}}_{a c}, \\
& f_{a b}+\sum_{c, d:(c, d) \in L} \overline{\mathbf{x}}_{a b}^{T} \mathbf{M}_{c d}^{a b} \overline{\mathbf{x}}_{c d} f_{c d} \leq 1
\end{aligned}
$$

\section{where}

$$
\begin{aligned}
\overline{\mathbf{x}}_{a b} \in\{0,1\}^{C}, \quad \overline{\mathbf{y}}_{a b} \in\{0,1\}^{I}, & f_{a b}>0, \\
& \forall a, b:(a, b) \in L .
\end{aligned}
$$

Intuitively, if a particular link is heavily loaded, its effective link-layer data rate should be increased by reducing the interfering links in its neighborhood. Note that problem (13) is a non-linear mixed-integer program and is not easy to solve. The terms $\overline{\mathbf{x}}_{a b}^{T} \overline{\mathbf{x}}_{a c}$ and $\overline{\mathbf{y}}_{a b}^{T} \overline{\mathbf{y}}_{a c}$ are quadratic and the term $\overline{\mathbf{x}}_{a b}^{T} \mathbf{M}_{c d}^{a b} \overline{\mathbf{x}}_{c d} f_{c d}$ is cubical.

\section{B. Equivalent Linear Problem}

By using some binary linearization techniques [11]-[13], the original non-linear mixed-integer problem in (13) can be converted to its exactly equivalent linear mixed-integer program. The following steps are required.

Step 1. For each pair of logical links $(a, b),(c, d) \in L$, we define a $C \times 1$ auxiliary vector $\overline{\mathbf{u}}_{c d}^{a b}$ to be as follows:

$$
\overline{\mathbf{u}}_{c d}^{a b}=\overline{\mathbf{x}}_{a b} \circ \overline{\mathbf{x}}_{c d}
$$

where $\circ$ denotes the Hadamard product ${ }^{1}$. From (14) we have, $\overline{\mathbf{x}}_{a b}^{T} \overline{\mathbf{x}}_{c d}=\mathbf{1}^{T} \overline{\mathbf{u}}_{c d}^{a b}$. Since $\overline{\mathbf{x}}_{a b}$ and $\overline{\mathbf{x}}_{c d}$ are $C \times 1$ binary vectors, eq. (14) is equivalent to the following linear constraint (see the Appendix):

$$
\begin{gathered}
\overline{\mathbf{x}}_{a b}+\overline{\mathbf{x}}_{c d}-\overline{\mathbf{u}}_{c d}^{a b} \leq \mathbf{1}, \\
\mathbf{0} \leq \overline{\mathbf{u}}_{c d}^{a b} \leq \overline{\mathbf{x}}_{a b}, \\
\mathbf{0} \leq \overline{\mathbf{u}}_{c d}^{a b} \leq \overline{\mathbf{x}}_{c d} .
\end{gathered}
$$

Step 2. For each pair of logical links $(a, b),(a, c) \in L$, we define an $I \times 1$ auxiliary vector $\overline{\mathbf{v}}_{a c}^{a b}$ to be as follows:

$$
\overline{\mathbf{v}}_{a c}^{a c}=\overline{\mathbf{y}}_{a b} \circ \overline{\mathbf{y}}_{a c} .
$$

From (16), we have, $\overline{\mathbf{y}}_{a b}^{T} \overline{\mathbf{y}}_{a c}=\mathbf{1}^{T} \overline{\mathbf{v}}_{a c}^{a b}$. Eq. (16) is equivalent to the following constraint (see the Appendix):

$$
\begin{gathered}
\overline{\mathbf{y}}_{a b}+\overline{\mathbf{y}}_{a c}-\overline{\mathbf{v}}_{a c}^{a b} \leq \mathbf{1}, \\
\mathbf{0} \leq \overline{\mathbf{v}}_{a c}^{a b} \leq \overline{\mathbf{y}}_{a b}, \\
\mathbf{0} \leq \overline{\mathbf{v}}_{a c}^{a b} \leq \overline{\mathbf{y}}_{a c} .
\end{gathered}
$$

Step 3. For each pair of logical links $(a, b),(c, d) \in L$, we define a $C \times C$ auxiliary matrix $\mathbf{Q}_{c d}^{a b}$ to be as follows:

$$
\mathbf{Q}_{c d}^{a b}=\overline{\mathbf{x}}_{a b} \overline{\mathbf{x}}_{c d}^{T} f_{c d}
$$

The entry in the $m^{t h}$ row and the $n^{t h}$ column of matrix $\mathbf{Q}_{c d}^{a b}$ is equal to the product of the $m^{t h}$ entry in vector $\overline{\mathbf{x}}_{a b}$ (a binary scalar), the $n^{t h}$ entry in vector $\overline{\mathbf{x}}_{c d}$ (a binary scalar), and $f_{c d}$ (a real scalar). Eq. (18) is equivalent to the following linear constraint for all $m, n \in\{1, \cdots, C\}$ (see the Appendix):

$$
\begin{gathered}
\mathbf{1}_{m}^{T} \mathbf{Q}_{c d}^{a b} \mathbf{1}_{n} \leq \mathbf{1}_{m}^{T} \overline{\mathbf{x}}_{a b}, \\
\mathbf{1}_{m}^{T} \mathbf{Q}_{c d}^{a b} \mathbf{1}_{n} \leq \mathbf{1}_{n}^{T} \overline{\mathbf{x}}_{c d}, \\
\mathbf{1}_{m}^{T} \overline{\mathbf{x}}_{a b}+\mathbf{1}_{n}^{T} \overline{\mathbf{x}}_{c d}-2+f_{c d} \leq \mathbf{1}_{m}^{T} \mathbf{Q}_{c d}^{a b} \mathbf{1}_{n}, \\
\mathbf{1}_{m}^{T} \mathbf{Q}_{c d}^{a b} \mathbf{1}_{n} \leq 2-\mathbf{1}_{m}^{T} \overline{\mathbf{x}}_{a b}-\mathbf{1}_{n}^{T} \overline{\mathbf{x}}_{c d}+f_{c d}
\end{gathered}
$$

where $\mathbf{1}_{m}$ denotes the standard basis vector (i.e., a $C \times 1$ constant vector with all entries equal to zero, except the $m^{\text {th }}$ entry which is equal to 1 ). The terms $\mathbf{1}_{m}^{T} \overline{\mathbf{x}}_{a b}, \mathbf{1}_{n}^{T} \overline{\mathbf{x}}_{c d}$, and $\mathbf{1}_{m}^{T} \mathbf{Q}_{c d}^{a b} \mathbf{1}_{n}$ simply denote the $m^{t h}$ entry of vector $\overline{\mathbf{x}}_{a b}$, the $n^{t h}$ entry of vector $\overline{\mathbf{x}}_{c d}$, and the entry in the $m^{t h}$ row and $n^{t h}$ column of matrix $\mathbf{Q}_{c d}^{a b}$, respectively. From (18), we have:

$$
\overline{\mathbf{x}}_{a b}^{T} \mathbf{M}_{c d}^{a b} \overline{\mathbf{x}}_{c d} f_{c d}=\mathbf{1}^{T}\left(\mathbf{Q}_{c d}^{a b} \circ \mathbf{M}_{c d}^{a b}\right) \mathbf{1} .
$$

Note that $\mathbf{M}_{c d}^{a b}$ is a constant matrix, thus eq. (20) is a linear equality in terms of $\mathbf{Q}_{c d}^{a b}$. In addition, we have, $\operatorname{tr}\left(\mathbf{Q}_{c d}^{a b}\right)=$ $\left(\mathbf{1}^{T} \overline{\mathbf{u}}_{c d}^{a b}\right) f_{c d}$.

\footnotetext{
${ }^{1}$ The Hadamard product of two $C \times C$ matrices $\mathbf{A}$ and $\mathbf{B}$ is a $C \times C$ matrix whose entry in the $i^{t h}$ row and $j^{t h}$ column is equal to the product of the entry in the $i^{t h}$ row and $j^{t h}$ column of $\mathbf{A}$ and the entry in the $i^{t h}$ row and $j^{\text {th }}$ column of $\mathbf{B}$. The Hadamard product of two $C \times 1$ vectors can be defined accordingly [14].
} 
Step 4. We first note that:

$\underset{\overline{\mathbf{x}}, \overline{\mathbf{y}}, f}{\operatorname{minimize}} \underset{a, b:(a, b) \in L}{\operatorname{maximum}} \frac{\gamma_{a b}}{f_{a b} c^{0}}=\underset{\overline{\mathbf{x}}, \overline{\mathbf{y}}, f}{\operatorname{maximize}} \underset{a, b:(a, b) \in L}{\operatorname{minimum}} \frac{f_{a b} c^{0}}{\gamma_{a b}}$

By defining $\delta$ as the minimum $\left(f_{a b} c^{0} / \gamma_{a b}\right)$ for all $(a, b) \in$ $L$, solving the right hand side in problem (21) is equivalent to maximizing $\delta$ subject to the constraint $\delta \leq\left(f_{a b} c^{0} / \gamma_{a b}\right)$ for all logical links $(a, b) \in L$.

Problem (13) can now be replaced by its equivalent linear mixed-integer program. Given $I, C, L, \gamma_{a b}, c^{0}$, and $\mathbf{M}_{c d}^{a b}$ for all $a, b, c, d \in N$ such that $(a, b),(c, d) \in L$,

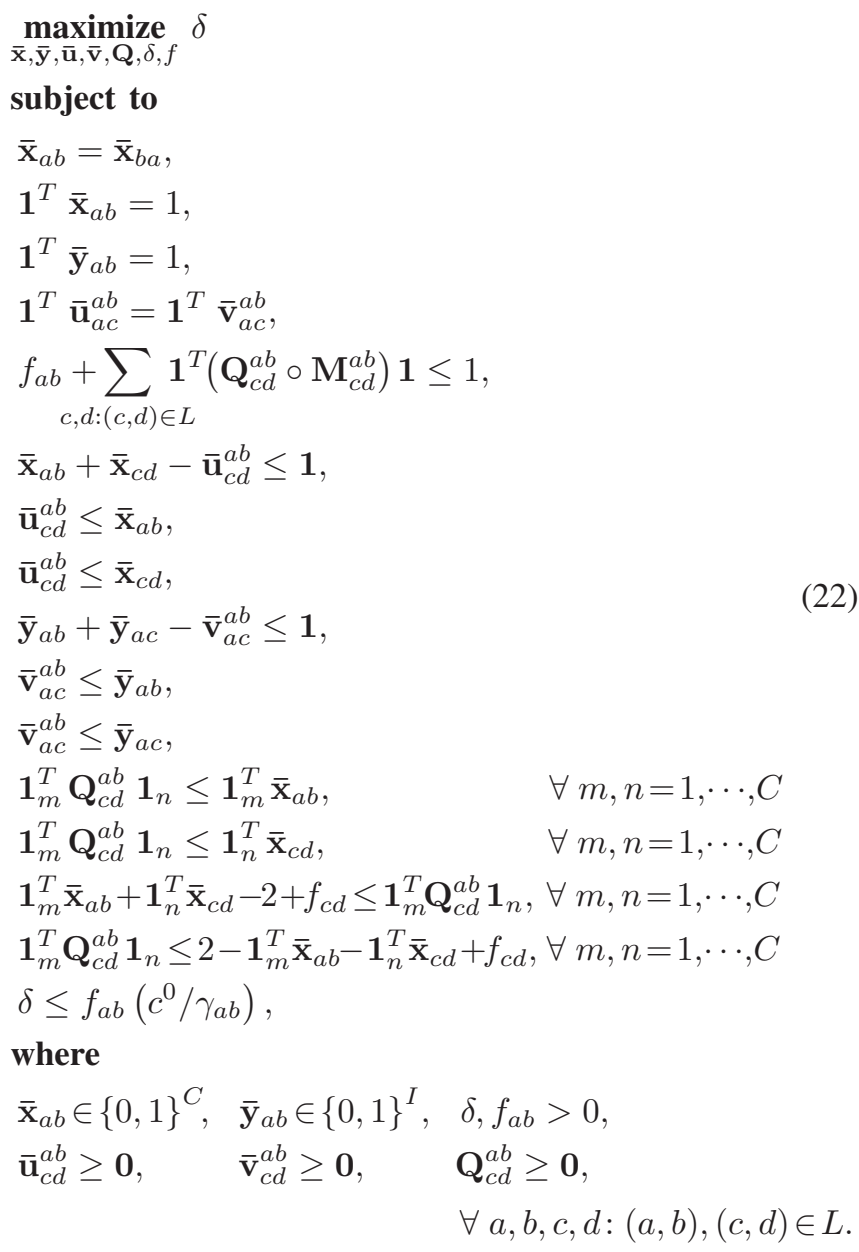

Note that the optimal solutions obtained from (13) and (22) are the same. There are effective commercial software (such as CPLEX [15]) to solve the linear mixed-integer programs. Most of them use branch-and-bound algorithm [16]. The linear mixed-integer problem (22) has $L(C+I)$ binary variables and $L^{2}\left(C+I+C^{2}\right)+L+1$ real variables, respectively. Note that the computational complexity of a linear mixedinteger program depends only on the number of its integer (in our case binary) variables, but not the number of real variables [7]. Thus, problem (22) can easily be solved in practice for small-scale and medium-scale MIMC-WMNs. Given the optimal solutions, the appropriate channels and interfaces can be assigned to the 802.11-based MIMC mesh routers. The optimal link-layer flow rates can be implemented by modifying the contention window adjustment mechanism within the 802.11 Distributed Coordination Function (DCF) as explained in [17].

\section{PERformance EVAluation}

In this section, we evaluate the performance gain when not only the non-overlapped channels, but also the partially overlapped channels are being used. In the simulation model, the size of the network field is $1 \mathrm{~km} \times 1 \mathrm{~km}$. Five different random scenarios are simulated. In each scenario, the WMN consists of 15 wireless mesh routers that are randomly located in the field. The routers are equipped with 3 NICs. (i.e., $I=3$ ). The IEEE 802.11 b with $11 \mathrm{Mbps}$ nominal data rate (i.e., $c^{0}=11 \times 10^{6}$ ) is being simulated. Thus, up to 11 partially overlapped frequency channels are available (see Fig. 2). Three of them (i.e., channels 1, 6, 11) are non-overlapped. The $S I N R_{\min }$ is set to be $13 \mathrm{~dB}$. The value of $\kappa$ is equal to 2 . The transmission power is the same for all routers. For each scenario, 30 source and destination pairs are randomly selected to generate UDP (User Datagram Protocol) traffic. We obtain the global optimal solution for linear mixed-integer program in (22) by using branch-and-bound algorithm [16]. To evaluate the performance, two metrics are considered: 1) aggregate network capacity (i.e., $\left.\sum_{a, b:(a, b) \in L} f_{a b}\right)$ and 2) bottleneck link utilization (i.e., $1 / \delta$ ).

The simulation results are shown in Fig. 4. In this figure, each point is the average of measurements for all five simulated scenarios. The dashed lines correspond to the measured performance metrics when a single channel (i.e., channel 1), two non-overlapped channels (i.e., channels 1, 6), and three non-overlapped channels (i.e., channels 1, 6, 11) are being used, respectively. It is observed that, by using the partially overlapped channels $1,2,3,4,5$, and 6 instead of using only non-overlapped channels 1 and 6 , the aggregate network capacity increases by $96 \%$ and the bottleneck link utilization decreases by $20 \%$. On the other hand, by using all partially overlapped channels $1,2, \cdots, 10$, and 11 instead of using only non-overlapped channels 1,6 , and 11, the aggregate network capacity increases by $93 \%$ and the bottleneck link utilization decreases by $50 \%$. Note that the performance improvements are achieved without using extra resources (frequency spectrum). Thus, the spectrum is utilized more efficiently when partially overlapped channels are being used.

\section{COnClusions}

In this paper, we proposed a joint channel assignment, interface assignment, and scheduling algorithm for MIMCWMNs when all non-overlapped and partially-overlapped channels are being used. The joint problem is formulated as a linear mixed-integer program with a few integer variables. The computational complexity is low and is feasible for implementation in practical networks. Simulation results show that there is a significant performance improvement in terms of a higher aggregate network capacity and a lower bottleneck link utilization when all the partially overlapped channels within the IEEE 802.11 b frequency band are being used. 


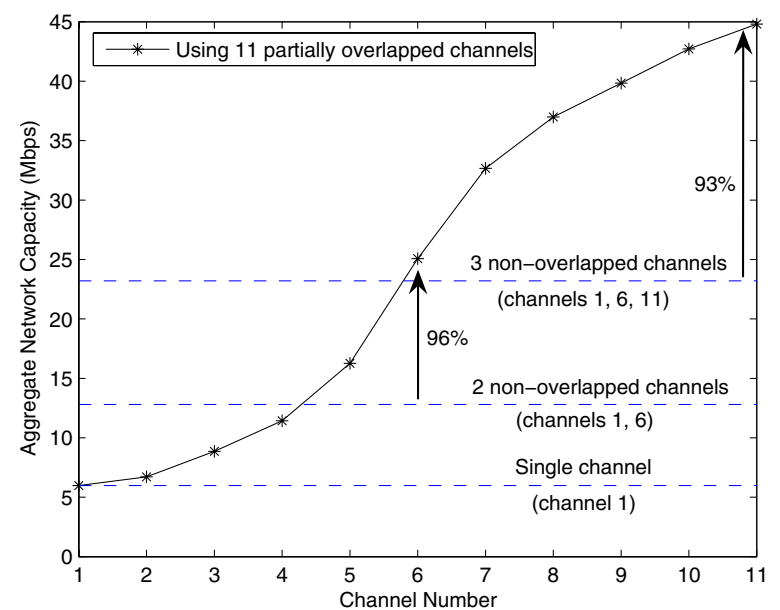

(a) Aggregate network capacity

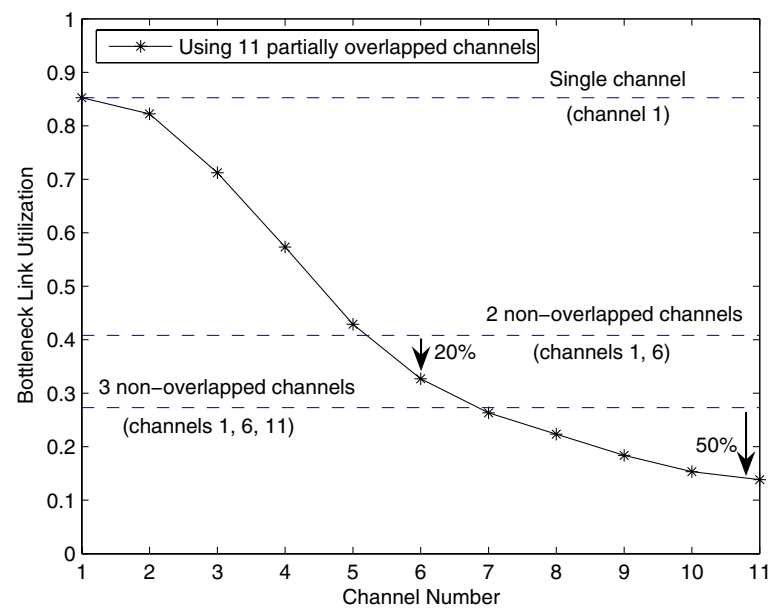

(b) Bottleneck link utilization

Fig. 4. Performance comparison within the IEEE $802.11 \mathrm{~b}$ frequency band.

For future work, we plan to extend our design by integrating logical topology design and routing algorithms. We shall also consider assigning more than one logical link between a pair of routers in order to increase the effective capacity.

\section{APPENDIX}

Consider two binary variables $\theta_{1}$ and $\theta_{2}$. We define $\pi=$ $\theta_{1} \theta_{2}$. That is,

$$
\pi= \begin{cases}0, & \text { if } \theta_{1}=0, \theta_{2}=0 \\ 0, & \text { if } \theta_{1}=0, \theta_{2}=1 \\ 0, & \text { if } \theta_{1}=1, \theta_{2}=0 \\ 1, & \text { if } \theta_{1}=1, \theta_{2}=1 .\end{cases}
$$

The desired correspondence is obtained by simply requiring that [11]:

$$
\begin{gathered}
\theta_{1}+\theta_{2}-\pi \leq 1 \\
0 \leq \pi \leq \theta_{1} \\
0 \leq \pi \leq \theta_{2}
\end{gathered}
$$

As an example, let $\theta_{1}=1$ and $\theta_{2}=0$. From (24) we have:

$$
\begin{gathered}
1+0-\pi \leq 1, \\
0 \leq \pi \leq 1, \\
0 \leq \pi \leq 0
\end{gathered}
$$

which means, $0 \leq \pi \leq 0$. In other words, we have, $\pi=0$.

Now consider a non-negative real variable $r$. Assume that $r_{\max }$ is an upper bound for the real variable $r$. We define $\sigma=$ $r \theta_{1} \theta_{2}$. The desired correspondence between $\sigma$ and variables $\theta_{1}, \theta_{2}$, and $r$ is obtained by simply requiring that [12]:

$$
\begin{aligned}
0 & \leq \sigma \leq r_{\max } \theta_{1}, \\
0 & \leq \sigma \leq r_{\max } \theta_{2}, \\
r_{\max }\left(\theta_{1}+\theta_{2}-2\right)+r & \leq \sigma \leq r_{\max }\left(2-\theta_{1}-\theta_{2}\right)+r .
\end{aligned}
$$

As an example, let $\theta_{1}=\theta_{2}=1$. From (26) we have, $r \leq$ $\sigma \leq r$. In other words, $\sigma=r$.

\section{ACKNOWLEDGMENT}

This work is supported by the Natural Sciences and Engineering Research Council of Canada (NSERC) under grant number 261604-03.

\section{REFERENCES}

[1] P. Bahl, A. Adya, J. Padhye, and A. Wolman, "Reconsidering wireless systems with multiple radios," ACM Computer Communication Review, vol. 34, pp. 39-46, Oct. 2004.

[2] A. Raniwala and T. Chiueh, "Architecture and algorithms for an IEEE 802.11-based multi-channel wireless mesh network," in Proc. of IEEE Infocom, Miami, FL, March 2005.

[3] M. Alicherry, R. Bhatia, and L. Li, "Joint channel assignment and routing for throughput optimization in multi-radio wireless mesh networks," in Proc. of ACM MobiCom, Cologne, Germany, Sept. 2005.

[4] A. H. Mohsenian Rad and V. W. S. Wong, "Joint optimal channel allocation, interface assignment, and MAC design for multi-channel wireless mesh networks," in Proc. of IEEE Infocom'07, Anchorage, AK, May 2007.

[5] — "Joint optimal channel assignment and congestion control in multiradio wireless mesh networks," in Proc. of IEEE ICC, Istanbul, Turkey, June 2006.

[6] A. Mishra, V. Shrivastava, S. Banerjee, and W. Arbaugh, "Partially overlapped channels not considered harmful," in Proc. of ACM SIGMetric, Saint Malo, France, June 2006.

[7] H. Taha, Operations Research: An Introduction, 7th ed. Prentice Hall, 2003.

[8] J. Proakis, Digital Communications, 4th ed. McGraw-Hill, 2000.

[9] A. H. Mohsenian Rad and V. W. S. Wong, "Logical topology design and interface assignment for multi-channel wireless mesh networks," in Proc. of IEEE Globecom, San Francisco, CA, November 2006.

[10] D. P. Bertsekas and R. Gallager, Data Communications, 2nd ed. Prentice Hall, 1992.

[11] F. Glover and E. Woolsey, "Further reduction of zero-one polynomial programming problems to zero-one linear programming," Operations Research, vol. 21, pp. 156 - 161, Jan. - Feb. 1973.

[12] C. T. Chang and C. C. Chang, "A linearization method for mixed 0-1 polynomial programs," Computers and Operations Research, vol. 27, pp. 1005 - 1016, Sept. 2000.

[13] W. Adams and H. Sherali, "A tight linearization and an algorithm for zero-one quadratic programming problems," Management Science, vol. 32, pp. 1274 - 1290, Oct. 1986.

[14] R. Horn and C. Johnson, Topics in Matrix Analysis. Cambridge University Press, 1994.

[15] "ILOG CPLEX." http://www.ilog.com/products/cplex/, 2006.

[16] A. H. Land and A. G. Doig, "An automatic method for solving discrete programming problems," Econometrica, vol. 28, pp. 497-520, 1960.

[17] Z. Fang and B. Bensaou, "Design and implementation of a MAC scheme for wireless mesh networks based on a cooperative game framework," in Proc. of IEEE ICC, Paris, France, June 2004. 